\title{
EINE NEUE LATEINAMERIKAPOLITIK DER VEREINIGTEN STAATEN?
}

Wieder einmal verkündet ein amerikanischer Präsident bestimmte Grundsätze für die Politik der Vereinigten Staaten gegenüber Lateinamerika. Plus ça change, plus c'est la même chose . . .? Am 31. Oktober 1969 hielt Präsident Nixon eine lange angekündigte, für das Verhältnis zu Lateinamerika programmatisch gemeinte Rede $^{1}$, deren Tenor eine deutsche Tageszeitung in der Formel zusammenfaßte: „USA geben Führungsrolle in Südamerika auf“2.

In seiner Ansprache ordnete Präsident Nixon die Diskussion einiger politischer und wirtschaftlicher Sachfragen dem Bemühen unter, das Verhältnis zu Lateinamerika generell $\mathrm{zu}$ definieren. Dieser Versuch folgt einem eindeutigen Trend im amerikanischen Bewußtsein: abzurücken von der allzu selbstgewiß und unaufgefordert übernommenen, weltweiten Aufgabe der Sicherung von „Freiheit" gegen "Aggression“ oder der Verwirklichung von sozial-politischem „Fortschritt“ (heute „Modernisierung“ genannt). Die frustrierenden Erfahrungen in Vietnam und im Dschungel der eigenen Megalopolis haben einen Prozeß der Selbstbefragung in Gang gebracht, dessen Ausmaß und Endpunkt noch ungewiß sind. Charakteristisch ist die kürzliche Äußerung eines jungen amerikanischen Historikers: "I hope we will go through a chastening period in which we will be knocked out of our grandiosity - a period in which we will see the self-righteous, illusory quality of that vision of ourselves offered by the high Washington official who said that while other nations have 'interests', the United States has 'a sense of responsibility'"3. Ungeachtet aller Theorien über die ökonomische Bedingtheit gerade der amerikanischen Politik ${ }^{4}$ werden die schwankenden außenpolitischen "moods" 5 ihren Eindruck auf einen Präsidenten Nixon angesichts seiner politischen Erfahrung, seines knappen Wahlsieges und der oppositionellen Mehrheiten im Kongreß nicht verfehlen ${ }^{6}$. Es ist schwer allgemein zu sagen, was der als außenpolitischer Ausdruck dieser introvertierten „withdrawal psychology"7 oft beschworene Neo-Isolationismus oder Revisionismus in der heutigen Situation konkret bedeuten kann - sicher nicht die Rückkehr zum „klassischen“ Isolationismus der Zwischenkriegszeit gegenüber anderen Industrienationen. Manche Beobachter fürchten jedoch eine völlige Interesselosigkeit („unconcern“) gegenüber den Problemen der Dritten Welt ${ }^{8}$.

Nixon sucht einen mittleren Weg. Er erklärt kurz und bündig: „We do care.“ Gleichzeitig trägt er der veränderten Stimmung Rechnung und führt damit das Grundthema weiter, das er bereits, weniger spektakulär gegenüber Europa, besonders nachdrücklich in Asien angeschlagen hatte und zuletzt in seiner großen Vietnam-Rede emphatisch bekräftigte: Entwicklung ebenso wie Sicherheit und Verteidigung seien Aufgaben der betroffenen Staaten selbst; die USA würden sie dabei

1 Vollst. Wortlaut in: Wireless Bulletin from Washington, ISIS, vom 3. Nov. 1969.

2 Die Welt vom 3. Nov. 1969

3 J. C. Thomson jr., No more Vietnams? Conference at the Adlai Stevenson Inst. of Int. Aff., June 1968, in: The Atlantic Monthly, Nov. 1968, S. 99-116 und Dez. 1968, S. 97-125, hier S. 125 . - Oder Under Secr. St. Richardson: "Americans are disillusioned by rhetoric, bored with false drama, and tired of exaggerated hopes and overblown undertakings.“ Anspr. 5. Sept. 1969 vor d. Am. Pol. Sc. Ass'n., Dpt. St. Bull. 61 (1969 II), S. 257.

4 Vgl. zuletzt noch als Aufbereitung von Marx und Beard im Hinblick auf Vietnam etwa G. Kolko, The roots of American foreign policy. An analysis of power and purpose, 1969; U. Küntzel, Der DollarImperialismus, 1968.

5 G. Almond, The American people and foreign policy (1950), S. 32.

6 Sein Under Secr. St. Richardson beschrieb diese harte Tatsache kürzlich mit gelungenem understatement als die "interaction between the philosophy and temperament of the man who is President and the mood, pressures, and requirements of the moment in history in which he leads the Nation ${ }^{\alpha}$, a. a. O. (Anm. 3), S. 257.

7 J. Reston: From leadership to partnership, Int. Herald Tribune v. 3. Nov. 1969.

8 E. Reischauer, Atlantic Monthly, Dez. 1968, S. 116, 122. 
- militärisch im Rahmen bestehender vertraglicher Pflichten - auf Ersuchen unterstützen; beide Aufgaben müßten aber stets in lokaler Verantwortung bleiben, die USA dürften sie nie als eigene übernehmen $-\ldots$. . to support the Asian countries' efforts to defend and develop themselves, without attempting to take from them the responsibilities which should be theirs", wie Nixon es in Bangkok formulierte ${ }^{9}$.

\section{II}

Auch jetzt gegenüber Lateinamerika gibt sich Präsident Nixon bewußt und absichtlich weniger grandios und prätentiös, weniger ideologisch-expansiv, selbstsicher und fortschrittsbewußt als es der liberal-missionarischen Tradition entspricht, in deren Sinne ein bekannter Politiktheoretiker (I. de Sola Pool) noch vor kurzem allzu flüssig formulieren konnte: es müsse das Ziel der USA sein, (to create) „a world in which the political systems of all states are democratic and pacifically oriented" ${ }^{10}$. Mit erstaunlichem Freimut erklärt dagegen Nixon, gelegentlich habe der Vorwurf überentwickelten Selbstvertrauens in die "rightness of our own prescriptions" durchaus getroffen; lange hätten die USA die Illusion gehegt, sie könnten "remake continents"; aus dem Bewußtsein ihrer Reichtümer und Technologie, aus ihren guten Absichten und ihrer üblichen Ungeduld heraus sowie unter dem Eindruck vom Erfolg des Marshall-Planes hätten sie „sometimes imagined that we knew what was best for everyone else and that we could and should make it happen. - But experience has taught us better."

Gewiß ist das zunächst nur eine rein verbale Abschwächung des traditionellen ideologisch-missionarischen Elementes amerikanischer Außenpolitik. Aber wenn der Präsident wiederholt betont, an die Stelle der von ihm kritisierten Haltung müsse eine "reifere Partnerschaft" im interamerikanischen System treten, welche die je eigenen Traditionen, den je eigenen Charakter der lateinamerikanischen Staaten zur Basis zu nehmen habe, so scheint sich darin doch ein neues Bewußtsein der "foreignness of foreigners" anzudeuten, dessen generelle Unterentwickeltheit zum Schaden der zwischenstaatlichen Beziehungen etwa St. Hoffmann als spezifisch amerikanischen Mangel denunziert ${ }^{11}$. Nixons angestrebter pragmatisch-unideologischer Ansatz - „no grandiose promises and no panaceas" - könnte in der Tat zunächst eine wichtige atmosphärische Wandlung bringen und dadurch auch konkrete Veränderungen ermöglichen. Was immer die USA tun oder nicht tun, hat in Amerika notwendig eine weitreichende, "führende“ Bedeutung. Die bloße Versicherung, $\mathrm{n}$ u $\mathrm{n} \mathrm{me} \mathrm{h} \mathrm{r}$ nur noch Partner unter Gleichen sein und das interamerikanische System entsprechend stärken zu wollen, klingt jedoch nicht unbedingt neuartig, nachdem diese Rolle bereits in Dutzenden von Konventionen, Resolutionen, Deklarationen usw. beschrieben und teilweise rechtlich bindend festgelegt worden ist.

Und am Ende, fast unbemerkt, verbindet sich der latente Führungsanspruch wieder mit dem traditionellen Missionsthema. Dabei erscheinen die lateinamerikanischen Staaten zunächst nicht mehr wie früher als Adressaten, vielmehr - sie haben es „nicht mehr nötig“ - als Träger dieses Missionsethos. Objekt ist nunmehr die extrakontinentale Welt. Das partnerschaftlich geeinte Amerika „will be an example for the world. Once again, by this example, we will stand for something larger than ourselves." Dieses "example"-Thema ist eines der Grundmotive des ameri-

9 Am 28. 7. 1969, Dpt. St. Bull. 61 (1969 II), S. 154; erste Formulierung in der Pressekonf. in Guam am 25. 7. 1969; aber auch gegenüber Nicht-Bündnispartnern wie Indien: "Asian problems must be resolved by the people of Asia. But we stand ready to help." Ibid., S. 158. "Wortlaut der Vietnam-Rede vom 3. Nov. 1969, Int. Herald Trib. v. 5. 11. 1969; ferner Under Secr. St. Richardson, ibid. (s. Anm. 3), S. $257-260$.

10 Atlantic Monthly, Nov. 1968, S. 114

$11 \mathrm{Vgl}$. St. Hoffmann, Gulliver's Troubles or the setting of American foreign policy (1968), Kap. 4-6. 
kanischen Selbstverständnisses ${ }^{12}$. Wieder die Unterordnung unter ein höheres, universal wenn nicht verpflichtendes, so doch zumindest relevantes Ideal. Nun bezeichnet Nixon Amerika zwar ausdrücklich als "community of widely diverse peoples“. Dieses Ideal der Partnerschaft der Verschiedenheiten als Modell einer Weltstruktur ist erkennbar die Projektion nordamerikanischer nationaler Erfahrung: der Bindung erheblicher nationaler, religiöser Verschiedenheiten durch einen allgemeinen Konsensus über eine (oft prozedurale) Wertbasis's. Wo nun der vorausgesetzte Konsensus in Amerika tatsächlich fehlt, dient das Verhalten der USA objektiv seiner Durchsetzung, ist es Forderung an Lateinamerika nach ideologisch fundierter „Partnerschaft" - kurz ideologische Mission (jetzt wieder mit Lateinamerika als Objekt). Das zeigt sich deutlich daran, daß ein radikales, antagonistisches Anderssein, das auch die Partnerschaft als Bindung ablehnt (etwa Kuba), mit ihr schlechthin unvereinbar ist. Nixon umgeht dieses Dilemma; Kuba läßt er unerwähnt.

III

Lediglich innerhalb dieser gemeinsamen Bindung visiert Nixon eine im Vergleich zur jüngeren Vergangenheit ideologisch weniger starre Haltung an, wenn er ankündigt, die USA würden bei aller Vorliebe für demokratische Prinzipien und Prozeduren „auf diplomatischer Ebene mit den Regierungen im interafrikanischen System realistischerweise so wie sie sind" verkehren müssen. Sie wollen also bei der immer wieder akuten Frage der Anerkennung von de facto-Regierungen vorerst keine demokratischen Legitimitäts- und verfassungsmäßigen Legalitätskriterien anwenden. Damit will Nixon der Tatsache Rechnung tragen, daß Instabilität und gewaltsame Regierungswechsel infolge der explosiven sozialen und politischen Spannungen in Lateinamerika vermutlich unvermeidbar sind. Das ist nun zwar eine ausdrückliche Abkehr von der stark ideologisch gefärbten Politik Kennedys, der die wirtschaftlichen Vorteile der Allianz für den Fortschritt an demokratisches und sozial-ökonomisches Wohlverhalten gebunden hatte: die Anerkennung von Umsturzregierungen und die Wiederaufnahme der bei einem Staatsstreich suspendierten Hilfsleistungen erfolgte erst nach bindenden Zusagen über die Rückkehr zu demokratischen Verfassungsgrundsätzen ${ }^{14}$. Präsident Johnson hatte damit tatsächlich schon im April 1964 gegenüber Brasilien gebrochen. Seither häufen sich Gelegenheiten, ähnlich zu verfahren. Aber nicht deshalb ist Nixons Ankündigung keine grundsätzliche Neuerung, sondern weil seine These nur älterer Tradition entspricht, die bis 1906/7 und von 1931/2 bis zum 2. Weltkrieg gegolten hatte. Dennoch ist für den Augenblick eine gewisse Entideologisierung der Lateinamerikapolitik abzusehen, wenn auch in den zuvor angedeuteten Grenzen und deshalb nicht frei von jenem fundamentalen Dilemma, nur mit Vorbehalt „realistisch“ sein zu können.

Die übrigen konkreten Ankündigungen (weitere Einzelheiten sollen einer späteren Konferenz des IA-ECOSOC unterbreitet werden) betreffen hauptsächlich wirtschaftliche Fragen: Handel und Entwicklungshilfe. Auch hier eine gewisse Pragmatisierung. Das ideologisch-ökonomische Allianz-Programm als Sondervorhaben wird praktisch ad acta gelegt. Beibehalten wird der allgemeine Wille zu helfen, wobei offen bleibt, ob aus dem "moral imperative" heraus oder weil man weiterhin - das war jedenfalls die Philosophie der "Allianz" - die Kausalreihe wirtschaftliches Wachstum/Modernisierung - Stabilität für richtig und die letzt-

$12 \mathrm{Vgl.} \mathrm{K.} \mathrm{Krakau,} \mathrm{Missionsbewußtsein} \mathrm{und} \mathrm{Völkerrechtsdoktrin} \mathrm{in} \mathrm{den} \mathrm{Vereinigten} \mathrm{Staaten} \mathrm{von} \mathrm{Amerika}$ (1967), S. $115 \mathrm{ff}$. u. passim.

$13 \mathrm{Vgl}$. St. Hoffmann (Anm. 11), S. 122 ff.; K. Krakau (Anm. 12), 1. Teil.

14 Vgl. Nachweise im einzelnen bei K. Krakau (Anm. 12), Kap. 13. 
genannte für lebenswichtig für die USA hält. Jedenfalls wird der Hilfswille praktisch sogleich dadurch erheblich qualifiziert, daß der außenpolitische Ausschuß des Abgeordnetenhauses aus Nixons schon reduziertem Lateinamerika-Hilfsprogramm ${ }^{15}$ für das nächste Jahr in Höhe von 437,5 Mill. $\$$ am Tage vor der Rede des Präsidenten 100 Mill. \$ herausstrich. Nixon hat keinerlei Widerspruch dagegen angemeldet. Noch unter Kennedy hatte es erbitterte Auseinandersetzungen mit dem Kongreß um diese Fragen gegeben. Weitere Abstriche sind im Bewilligungsverfahren mit Sicherheit zu erwarten.

Bedeutsam ist aber die grundsätzliche Bereitschaft der Regierung, die amerikanische Hilfe im Rahmen des interamerikanischen Systems auf eine multilaterale Basis zu stellen und sie als nordamerikanische Unterstützung für lateinamerikanische Initiativen zu konzipieren. Nixon kommt damit Forderungen entgegen, die nicht nur in Lateinamerika, sondern auch in den USA immer wieder erhoben worden sind: um die vielfältigen psychologischen, ökonomischen und politischen Belastungen und die besondere Interventionsgefahr, die sich aus der bilateralen Hilfsbeziehung ergeben, abzuschwächen, sollten die Hilfsmechanismen internationalisiert sowie ferner die Planung und Ausführung völlig von der Bank-Funktion getrennt werden. Auf die letztere, d. h. das Angebot von Materialien und Kapital, auch zum Zwecke der Engagierung kompetenter Planer und Verwalter, sollten sich die Geberländer beschränken. Planung und Ausführung sollten ausschließlich der Kontrolle der Empfängerländer überlassen bleiben ${ }^{16}$. So weit geht indessen der Vorschlag des Präsidenten nicht. Danach soll zwar ein interamerikanisches Organ, etwa der Interamerikanische Ausschuß für die Allianz für den Fortschritt (CIAP) oder ein neu zu schaffendes Gremium, einen wachsenden Teil der Verantwortung für Entwicklungshilfeentscheidungen übernehmen; später einmal soll dieses Organ „major operational and decision-making responsibilities" erhalten. Etwas vage bleibt indessen die konkrete Aufgabe. Sie soll offenbar im Bereich der Planung und Durchführung liegen, denn es heißt weiter, die lateinamerikanischen Nationen würden dadurch eine Hauptrolle spielen ,in setting priorities within the hemisphere, in developing realistic programs, and in keeping their own performance under critical review". In zwei wichtigen Punkten jedoch bleibt dieses Programm hinter den Forderungen zurück. Erstens ist die Trennung zwischen Planung/Durchführung und Finanzierung keineswegs vollständig. Die USA würden vermutlich in diesem Gremium in Zukunft ebenfalls vertreten sein und ihre Stimme wird unvermeidbar schwerer wiegen als die der Partner. Deren Konzeptionsautonomie ist also sogleich wieder in Frage gestellt. Zum anderen wird der Hilfsmechanismus selbst (Verteilung von Entwicklungsmitteln) nicht multilateralisiert. Lediglich Leitlinien, Schwerpunkte, auch konkrete Entwicklungsprogramme können die Lateinamerikaner gemeinschaftlich (und auch das vermutlich nur gemeinsam mit den USA) definieren und ausarbeiten. Die Hilfsvereinbarung als solche bleibt bilateral und damit jedes Programm, auch wenn es auf einer Kollektiventscheidung des CIAP beruht, doch letztlich dem nordamerikanischen Veto unterworfen. Denn die USA mögen zwar einen Beschluß des CIAP nicht ändern können. Niemand kann sie aber zwingen, auf der Basis dieses Beschlusses überhaupt ein zweiseitiges Hilfsabkommen zu schließen. Es wird also nicht ein - und sei es noch so kleiner - gemeinschaftlicher Entwicklungsfonds gemeinschaftlich verwaltet und verteilt werden. Das deutet auch Präsident Nixon selbst an, wenn er von dem Ziel spricht, „an effective multilateral framework for bilateral assistance" $\mathrm{zu}$ entwickeln. Immerhin würden die Funktionen des neuen CIAP im Sinne echter eigener Planungs- und Kontroll-

$15 \mathrm{Vgl}$. die Kongreßbotsch. d. Präs. betr. das Auslandshilfsprogramm 1970/71, v. 28. 5. 1969, Dpt. St. Bull. 60 (1969 I), S. $515 \mathrm{ff}$

16 Vgl. z. B. J. W. Fulbright, The arrogance of power (1966), S. $223 \mathrm{ff}$.; E. Reischauer, J. C. Thomson jr., Atlantic Monthly, Dez. 1968, S. 112, 115, 118; Erkl. d. Mitgliedstaaten d. Sonderkom. f. lateinamerik. Koordination (CECLA) der OAS von Viña del Mar/Chile v. 17. 5. 1969, Europa Archiv 20 (1969) D 488 unter Ziff. $28 \mathrm{f}$. 
befugnisse über seinen bisherigen Aufgabenbereich hinausgehen, der sich auf Koordination, Vermittlung, Begutachtung und Beratung, also bilaterale Beziehungen, beschränkte.

Im Zusammenhang mit der Entwicklungshilfe fiel Nixon übrigens noch einmal in die ideologische Tonart zurück, als er - unter Berufung auf die eigene nationale Erfahrung - besonders emphatisch die Rolle privaten Kapitals, privater Investitionen und Unternehmen im Entwicklungsprozeß hervorhob. Er blieb dabei nicht nur theoretisch, sondern versprach, mit Entwicklungsmitteln nachdrücklich private Unternehmen der Empfängerländer zu fördern.

Ein weiterer oft kritisierter Aspekt der nordamerikanischen Entwicklungshilfe ist deren Bindung an die Verpflichtung, gewährte Darlehen für industrielle Erzeugnisse oder Dienstleistungen in den USA auszugeben, auch wenn die Weltmarktpreise für entsprechende Wirtschaftsgüter niedriger sind. Das mindert die Substanz der Entwicklungshilfe und führt andererseits dazu, daß, wie nicht ganz zu Unrecht behauptet wird, die Entwicklungshilfe der nordamerikanischen Industrie mehr hilft als den Entwicklungsländern. Präsident Nixon gibt nun ab sofort Entwicklungsgelder zu beliebigen Einkäufen auch in Lateinamerika frei. In Drittländern dürfen diese Entwicklungsgelder jedoch auch weiterhin nicht ausgegeben werden. Wenn es auch vom amerikanischen Standpunkt aus verständlich ist, daß man mit der völligen Freigabe nicht gerne der europäischen oder japanischen Konkurrenz aufhelfen möchte, so erweist sich dieses „Zugeständnis“ für die amerikanischen Entwicklungsländer aber doch als optische Täuschung, denn in Lateinamerika sind die in erster Linie benötigten Investitionsgüter und sonstigen Industrieerzeugnisse oft genug nicht vorhanden ${ }^{17}$. Weitere Bindungen dieser Art will Nixon überprüfen lassen.

Darin erschöpfen sich die konkreten Angebote an Lateinamerika bereits, sofern man in den beschriebenen Maßnahmen jedenfalls einen positiven ersten Schritt in der gewünschten Richtung sehen will.

\section{VII}

Das andere große Problem Lateinamerikas wie aller Entwicklungsländer sind die direkten und indirekten Handelsbeschränkungen zwischen ihnen und den Industriestaaten: Zölle, Mengenbegrenzungen, Vorzugssysteme, Ungleichgewichtigkeiten zwischen den Preisen für Rohstoffe und Fertigprodukte usw. Diese Schwierigkeiten zu mindern ist um so dringender, je geringer das Volumen der Entwicklungshilfe wird.

Präsident Nixon ist die Problematik dieser Frage durchaus bewußt. Seine Heilungsvorschläge erweisen sich jedoch als bloße Verschleierung des Nichtwillens oder Unvermögens, konkret irgend etwas zu tun, wenn er sich hinter die gleiche Lage a 11 e r Entwicklungsländer zurückzieht. Die Absichtserklärung, das Los der gesamten Dritten Welt insofern bessern zu helfen - was nur unter Mitwirkung aller Industrienationen möglich wäre -, kommt der Ankündigung gleich, nichts tun zu wollen. Nixon erklärt, er werde sich bemühen, die nicht in Zöllen bestehenden Handelsschranken fast aller Industriestaaten im Verhältnis zu Lateinamerika und anderen Entwicklungsländern abbauen sowie ein liberales allgemeines System von Zollpräferenzen zugunsten a 11 e r Entwicklungsländer einführen zu helfen; ferner

17 Die Beschlüsse von Viña del Mar (Anm. 16), Ziff. 29, betrachten dies denn auch ausdrücklich nur als „mögliche Ưbergangslösung ${ }^{\alpha}$. 
wolle er den gleichen Zugang für alle Entwicklungsländer zu den Industriemärkten anstreben, um gewisse Diskriminierungen gegenüber Lateinamerika zu eliminieren - eine diskrete Anspielung auf die Assoziierungspolitik der EWG. Hier wird zwar ein Problem diagnostiziert, aber keine Remedur geboten. Die Reaktion ist wohlwollendes Wunschdenken, wie man etwa die Sicherheitsfrage mit dem Vorschlag einer Weltregierung mit Gewaltmonopol zu „lösen“ glaubt. - Ähnlich besteht die Antwort auf das Problem des wachsenden Schuldendienstes, der die weitere Entwicklung zunehmend gefährdet, in dem Rat, das CIAP möge die internationalen Finanzorganisationen drängen, mögliche Schritte zu empfehlen, geht mithin über ein verständnisvolles Achselzucken nicht hinaus. Die von fast allen nordamerikanischen Regierungen bei passenden Gelegenheiten wiederholte Versicherung hier zuletzt durch Secretary of State Rogers -, daß im Vergleich zu Lateinamerika "there is no part of the world that is more important to us"18, bleibt im Rahmen traditioneller Rhetorik $\mathbf{1 8 a}$.

Weiter erklären die USA sich bereit, Lateinamerika in allen diesen Fragen zu konsultieren; im interamerikanischen System solle ein prozeduraler Rahmen dafür geschaffen und genutzt werden, b e v or handelspolitische Maßnahmen getroffen würden. Auch wollten die USA ihre Wirtschaftspolitik, soweit sie Lateinamerika betrifft, der Beurteilung durch das CIAP zugänglich machen. Schließlich solle ein Under Secretary of State for Inter-American Affairs statt des bisherigen Assistant Secretary alle die Hemisphäre betreffenden staatlichen Aktivitäten koordinieren. Dieser letzte Punkt ist natürlich eine nur optische Aufwertung der Belange des Kontinentes.

Die Verweisung auf die Konsultationstechnik projiziert wieder ein wichtiges amerikanisches ideologisches Stilement auf die interamerikanischen Verhältnisse. „Konsultation" ist in einer durch weitgehenden ideologischen Konsensus geeinten Gesellschaft, die keine fundamentalen Konflikte kennt, eine geeignete Technik zur Bewältigung sekundärer technischer Differenzen. Sie konzentriert sich auf Fragen der Prozedur, Technik, Effizienz zur bestmöglichen Realisierung des vorgegebenen ideologischen Grundmusters (social engineering) ${ }^{19}$. Dieses Konfliktlösungsmodell paßt aber nicht für zwischenstaatliche Konfliktsituationen mit verschiedenartigen nationalen Stilen und existentiell antagonistischen Interessen wie im handels- und allgemein politischen Bereich in Amerika. Natürlich sind Konsultationen im internationalen Verkehr nicht per se schädlich - im Gegenteil sind sie notwendige Voraussetzung für jeden Konfliktausgleich und damit für Politik schlechthin. Aber das von dem spezifisch nordamerikanischen Verständnis getragene Modell "Konsultation" trägt nichts zur Lösung konkreter internationaler Konflikte bei, solange die USA nicht zu grundsätzlichen Zugeständnissen und Kompromissen bereit sind. Dann aber sind Konsultationen um ihrer selbst willen bestenfalls überflüssig, möglicherweise sogar schädlich, weil sie als solche schon eine "Lösung" vorspiegeln und dadurch die Suche nach echtem politischem Ausgleich nur erschweren.

\section{VIII}

Schließlich versichert der Präsident die Bemühungen um regionale Wirtschaftsintegration in Lateinamerika der Unterstützung durch die USA, soweit sie gewünscht werde. Im Gegensatz zu früheren amerikanischen Bekundungen, ja einer von den

\footnotetext{
18 Pressekonf. am 5. 6. 1969, Dpt. St. Bull. 60 (1969 I), S. 530.

18a Möglicherweise ist diese Beurteilung doch zu pessimistisch. Präsident Nixon gab inzwischen bekannt, die USA hätten die übrigen Industrienationen über die OECD bereits aufgefordert, Zollpräferenzen für a $11 \mathrm{e}$ Entwicklungsländer zu beschließen; geschehe das nicht in angemessener Zeit, würden die USA einseitig zugunsten Lateinamerikas allein handeln. (Allerdings müßte der Kongreß zustimmen, in dem erhebliche Widerstände zu erwarten sind.) Außerdem prüften die USA schon konkret die Möglichkeiten, die lateinamerikanischen Auslandsschulden gegenüber den USA zu refinanzieren, nach Int. Herald Trib. v. 12. 11. 1969

19 Ausführlich St. Hoffmann (Anm. 11), S. $122 \mathrm{ff} ., 194 \mathrm{ff}$.
} 
USA bewußt propagierten Integrationsideologie, enthält sich Nixon jedoch jeder positiven Bewertung dieser Integrationsversuche. Diese Zurückhaltung geht möglicherweise auf den Bericht des Gouverneurs Rockefeller über seine Lateinamerikareisen zurück, der zwar bisher nicht veröffentlicht wurde, über den aber gleichwohl einiges bekannt geworden ist ${ }^{20}$. Rockefeller spricht sich darin gegen eine Integration aus, die nur auf gegenseitigen Zollsenkungen beruht, wenn nicht gleichzeitig sich wechselseitig ergänzende industrielle Strukturen geschaffen werden. Sonst würden die durch die Handelsliberalisierung vergrößerten Wirtschaftsräume in erster Linie wieder den übermächtigen nordamerikanischen Unternehmen einschließlich ihrer lateinamerikanischen Dependencen zugute kommen, die automatisch diesen Markt beherrschen würden. Industrialisierung gehöre also zu einem sinnvollen Integrationskonzept. Dieselben Erwägungen, wenn auch gar nicht einmal mit ausdrücklicher Spitze gegen die USA, liegen auch den lateinamerikanischen Bemühungen um subregionale Integration zugrunde ${ }^{21}$. Nur ist die dort gezogene Folgerung nicht: weniger, sondern: bessere Integration. In Nixons Äußerungen kommt Rockefellers These wie gesagt abgeschwächt zum Ausdruck. Er wollte den Glauben an das Allheilmittel Integration wohl nicht verletzen.

\section{IX}

Obwohl das Programm des Präsidenten offenbar in großen Zügen den Empfehlungen des Gouverneurs folgt, sind doch gewisse Abweichungen bedeutsam. So wollte Rockefeller offenbar noch eindeutiger als Nixon die Rolle der USA auf diejenige des interessierten Freundes und Bankiers reduziert sehen. Ein noch interessanterer Punkt - und insofern ist die Botschaft des Präsidenten wichtig auch durch das, was sie nicht sagt - betrifft den Komplex Enteignungen. Dieses Thema ist seit einem Jahr angesichts der Ereignisse in Peru und jüngst in Bolivien wieder akut. Gegenüber Peru hat sich die nordamerikanische Regierung fast krampfhaft bemüht, jede Zuspitzung zu vermeiden und die hinhaltende Taktik Perus als "geeignete Schritte“ zur Regelung der Entschädigungsfrage im Sinne der amerikanischen Gesetzgebung zu interpretieren, um die von dieser angedrohten Gegenmaßnahmen - Einstellung der Wirtschaftshilfe, sog. Hickenlooper Amendment - zu vermeiden. Der Fall Bolivien erscheint bis jetzt als Schulbuchillustration des Vulgärmarxismus: eine amerikanische Ölgesellschaft wird verstaatlicht, die etwaige Entschädigung soll sich auf den Kapitalwert der Anlagen und Ausrüstungen beschränken ${ }^{22}$, wäre also nicht prompt, adäquat und effektiv nach amerikanischer Vorstellung; daraufhin verlangt die Gesellschaft vom State Department nicht diplomatischen Schutz, sondern Sanktionen gegen den Rechtsverletzer ${ }^{23}$. Die Regierung wartet auch hier vorläufig ab. Gouverneur Rockefeller empfahl nun, in diesen Fällen auf die Anwendung punitiver Sanktionen ganz zu verzichten. Das ist eine konsequente Fortführung der gegenwärtigen Zurückhaltung gegenüber dem Wandlungsprozeß in Lateinamerika, wie sie schon in der Anerkennungsfrage zum Ausdruck gekommen war, führt aber doch darüber hinaus. Rockefeller will nicht nur Widersprüche zur propagierten eigenen Ideologie, d. h. mittelbare Interessenverletzungen, sondern sogar die direkte Beeinträchtigung bedeutender ökonomischer (auch höchstpersönlicher) Rechte hinnehmen. Aber selbst wenn die Regierung von der Richtigkeit dieses Standpunktes überzeugt sein sollte - und dafür spricht ihr tatsächliches Verhalten -, so erschien es dem Präsidenten minde-

20 Bericht in Internat. Herald Trib. v. 30. 10. 1969. - Er ist inzwischen veröffentlicht worden, s. Int. Herald Trib. v. 12. 11. 1969, liegt aber noch nicht vor.

21 Vgl. G. Valdés, Der Anden-Vertrag. Ein neuer Ansatz zur wirtschaftlichen Integration in Lateinamerika, Europa Archiv 20 (1969), S. 699 ff.

$22 \mathrm{Vgl}$. Le Monde v. 21. 10. 1969.

23 Nach Int. Herald Trib. v. 31. 10. 1969. Die amerikan. Gesellschaft wendet dagegen ihre eigenen Sanktionen an: sie transportiert und vertreibt das exportfähige Ol nicht mehr, Le Monde v. 26. 10.1969. 
stens zu riskant, sich öffentlich dazu zu bekennen: einmal der inneramerikanischen Offentlichkeit und dem eigenen Gesetzgeber gegenüber; und in Lateinamerika dürfte eine solche offizielle Erklärung des Präsidenten der Vereinigten Staaten der sicher ohne Widerspruch bleibenden Aufforderung gleichkommen, nach Belieben mit amerikanischen Investitionen (dem größten Anteil aller Auslandsinvestitionen der USA) zu verfahren. Das schließt zwar eine an diesem Gedanken orientierte vorsichtige Politik nicht aus. Diese wird aber auf absehbare Zeit noch nicht davon überzeugt sein, daß es ihr "first imperative (sei) that the world must be made safe for revolution" 24 .

$$
\mathrm{X}
$$

Haben also die USA ihre „Führungsrolle aufgegeben“? Die vorangegangenen kurzen Ausführungen deuten die Vielschichtigkeit der Probleme an. Wenn die Position, von der aus die USA in der Vergangenheit in vielfältiger Weise die Entwicklungen in Lateinamerika - ob zum Guten oder Schlechten - beeinflußt haben, eine "Führungsrolle" ist, dann scheinen sich eine vorsichtige Abwendung von allzu direkter und oft überheblicher Ideologiehaftigkeit und darauf beruhender Einflußnahme, statt dessen eine pragmatischere Einstellung und Zurückhaltung anzukündigen. $\mathrm{Ob}$ diese Tendenz den Test unvorhergesehener (revolutionärer) Entwicklungen bestehen wird, bleibt abzuwarten.

Knud Krakau

24 R. J. Barnet, Intervention and revolution. The United States in the third world (1968), S. 283. 